\title{
Stochastic Safety for Random Dynamical Systems
}

\author{
Manuela L. Bujorianu ${ }^{1}$, Rafał Wisniewski ${ }^{2}$, Evangelos Boulougouris ${ }^{1}$
}

\begin{abstract}
In the paper, we study the so-called p-safety of a random dynamical system. We generalize the existing results for safety barrier certificates for deterministic dynamical systems and Markov processes. Moreover, we consider the case of random obstacles, modelled as random sets. This leads to the necessity of using integrals with respect to lower and upper distributions. We prove that if there exists at least one barrier certificate then the random dynamical system is safe. The barrier certificates are also defined using such nonlinear distributions. Furthermore, when the family of stochastic Koopman operators has the semigroup property, the barrier certificates are solutions for some type of Dirichlet problems.

Keywords: random dynamical system, Koopman operator, psafety, random set, occupation measure, hitting measure, supermedian function, barrier certificates.
\end{abstract}

\section{INTRODUCTION}

In this paper, we broaden our studies on stochastic safety verification via barrier certificates [4], [15], [16] considering the case of random dynamical systems. These are dynamical systems, in which the motion equation is randomized. The theory of random dynamical systems unites and develops the classical deterministic theory of dynamical systems and probability theory, with many applications in a very wide range of disciplines from physics to engineering, finance, or economics.

The results, presented here, can be instantiated for safety of deterministic dynamical systems and Markov models. The safety barriers are defined as elements of the class of supermedian functions, which are related to Lyapunov functions. Moreover, in this paper, we extend our investigations considering random obstacles that will be modelled as random sets. Modelling unsafe regions using random sets has been used in different applications, for example in air traffic management - for the random spread of winds and storms [14], in fire safety - for description of the process of accidental spread of a forest fire [18], and so on.

Formally, we define the concept of stochastic safety for random dynamical systems, as a sort of reach avoidance problem. The forbidden set is modelled by a random set, which is defined as a set-valued random variable. As in the case of Markov processes [4], we define hitting times, occupation and hitting measures for random dynamical systems. These will be employed in the description of random safety. Then we extend the concept of barrier certificate from [16] to make it able to deal with random obstacles. When defining

\footnotetext{
1 Maritime Safety Research Center, Department of Naval Architecture, Ocean \& Marine Engineering, University of Strathclyde, Scotland, UK \{Luminita.Bujorianu, Evangelos.Boulougouris\}estrath.ac.uk

2 Department of Electronic Systems, Automation and Control, Aalborg University, Denmark, raf@es.aau.dk
}

the new type of barrier certificates, the difficulty that arises is the need to use nonlinear integrals with respect to the lower or upper distributions of random sets. Then for proving further properties, we use the probability distributions of the random selectors for the underlined random sets.

For the given random dynamical system, we define two types of Koopman operators: random and stochastic. The family of random Koopman operators has always the semigroup property (derived from the definition of random dynamical system). But, the family of stochastic Koopman operators has the semigroup property only if the underlying dynamical system is Markovian. If this is true, we prove that the safety function is solution for a nonstandard Dirichlet problem associated to the infinitesimal generator of the stochastic Koopman semigroup.

The problem of stochastic safety verification using barrier certificates has been initiated in [12]. The overarching goal of barrier certificates of dynamical systems is to ensure that the dynamical system does not violate constraints. In the Markovian case (and the more general RDS case) this corresponds to the stability of the density dynamics, the control of which is mandated via optimal control [6]. Recently, the optimal control related Hamilton-Jacobi-Bellman theory has also been used in combination with barrier certificate methods to address robust and safe operation of stochastic systems [19].

The paper is structured as follows. In Section II, we give the main definitions for random dynamical systems and their random Koopman operators. As well, we define the concept of random hitting time, which will be later used in the definition of safety. In Section III, we generalize the stochastic safety to random dynamical systems with random obstacles, modelled as random sets. Then, using generalized distributions of random sets, we define the occupation measure and the hitting measure associated to the random safety problem. The connection between these measures is also discussed. Section IV comprises the main contribution of this paper. First, we define the concept of barrier certificate for random safety. Then, we prove in theorem 1 that if a random dynamical system has a barrier certificate, then it is safe. In this section, we refine the concept of Koopman operators introducing stochastic Koopman operators. To obtain further results, we suppose that the family of stochastic Koopman operators has the semigroup property. Under this assumption, we prove in Theorem 2 that the safety function is a solution of a nonlinear Dirichlet problem. In this setting, non-linearity does not refer to the Laplace operator (in our case, we work with the infinitesimal generator of the stochastic Koopman operator semigroup), but to the integration with respect to 
the generalized distributions of random sets. In the Section $\mathrm{V}$, we present some standard examples of random dynamical systems and sketch the methods to design barrier certificates. The paper ends with some conclusions.

\section{NotATION}

$\mathbb{R}_{+} \equiv\{x \in \mathbb{R} \mid x \geq 0\}$. The complement of a set $D$ is denoted by $D^{c}$. $I_{D}$ denotes the indicator function of $D$, i.e., $I_{D}$ is 1 on $D$, and 0 on its complement $D^{c}$.

The Borel sigma-algebra on a topological space $\mathcal{Y}$ is denoted by $\mathcal{B}(\mathcal{Y})$. In this paper, $\mathcal{Y}$ will be a Polish space that will be the state space for the given dynamical system. $\mathcal{B}^{b}(\mathcal{Y})$ will be the Banach space of bounded measurable real-valued functions on $\mathcal{Y}$, equipped with the supremumnorm. We will denote by $(\Omega, \mathcal{F}, \mathbb{P})$ the reference probability space. We denote by $\mathbb{E}$ the expectation corresponding to a probability $\mathbb{P}$.

By a cone, we understand a set $\mathcal{C}$ that satisfies 1) $\mathcal{C}+\mathcal{C} \subseteq$ $\mathcal{C}$, 2) $\left.\mathbb{R}_{+} \mathcal{C} \subseteq \mathcal{C}, 3\right) \mathcal{C} \cap(-\mathcal{C})=\{0\}$.

\section{RANDOM DYNAMICAL SySTEMS}

\section{A. Definitions}

In this subsection, we briefly present the main definitions for random dynamical systems (RDS) (see, e.g. [1]). A continuous RDS comprises two components:

1) A metric dynamical system $\theta$ defined on $(\Omega, \mathcal{F}, \mathbb{P})$, which is given as a mapping $\theta: \mathbb{R} \times \Omega \rightarrow \Omega$ such that

$$
(t, \omega) \mapsto \theta_{t}(\omega):=\theta(t, \omega)
$$

and the following conditions are satisfied:

- shift condition: $\theta_{s+t}=\theta_{s} \circ \theta_{t}, \theta_{0}=I_{\Omega}$, for any $t, s \in \mathbb{R}$;

- measurability: $(t, \omega) \mapsto \theta(t, \omega)$ is measurable w.r.t. both arguments;

- $\theta$-invariance: $\mathbb{P}\left(\theta^{-1}(F)\right)=\mathbb{P}(F)$ for any $t \in \mathbb{R}$ and $F \in \mathcal{F}$.

2) A cocycle $\phi$ on $\mathcal{Y}$ over $\theta$, which is given as a mapping $\phi: \mathbb{R} \times \Omega \times \mathcal{Y} \rightarrow \mathcal{Y}$ such that

$$
(t, \omega, x) \mapsto \phi(t, \omega, x)
$$

and the following conditions are satisfied:

- measurability: $(t, \omega, x) \mapsto \phi(t, \omega, x)$ is measurable;

- continuity: for each $\omega \in \Omega$, the mapping $(t, x) \mapsto$ $\phi(t, \omega, x)$ is continuous;

- cocycle equation: the family of random maps $\phi_{t}(\omega):=\phi(t, \omega, \cdot): \mathcal{Y} \rightarrow \mathcal{Y}$ satisfies the cocycle equations, i.e.,

$$
\phi_{s+t}(\omega)=\phi_{s}\left(\theta_{t} \omega\right) \circ \phi_{t}(\omega), \phi_{0}(\omega)=I_{\mathcal{Y}}
$$

The RDS is denoted by $(\theta, \phi)$. It is possible to associate to it, the skew product defined as a mapping $\Phi: \mathbb{R} \times \Omega \times \mathcal{Y} \rightarrow$ $\Omega \times \mathcal{Y}$, with $(t, \omega, x) \mapsto \Phi_{t}(\omega, x)$ given by:

$$
\Phi_{t}(\omega, x):=\left(\theta_{t}(\omega), \phi_{t}(\omega) x\right)
$$

The skew product is the main tool used to generalize the concepts from deterministic dynamical systems to RDS.

\section{B. Random Koopman operators}

Let $(\theta, \phi)$ be a continuous RDS. The random Koopman operator semigroup is defined by:

$$
U_{t} f(\omega, x):=f\left(\Phi_{t}(\omega, x)\right)=f\left(\theta_{t} \omega, \phi_{t}(\omega) x\right),
$$

for all $f \in \mathcal{B}^{b}(\Omega \times \mathcal{Y}), \omega \in \Omega, x \in \mathcal{Y}$. Note that the family of random Koopman operators is, in fact, associated to the skew product (1), which is a dynamical system. Therefore, the semigroup property holds. The associated random potential operator is defined by:

$$
G f(\omega, x):=\int_{0}^{\infty} U_{t} f(\omega, x) d t,
$$

for all $f \in \mathcal{B}^{b}(\Omega \times \mathcal{Y}), \omega \in \Omega, x \in \mathcal{Y}$.

The cone of supermedian functions associated to the semigroup $\mathcal{U}:=\left(U_{t}\right)_{t \geq 0}$ is defined as:

$$
\mathcal{S}_{\mathcal{U}}:=\left\{u \in \mathcal{B}^{b}(\Omega \times \mathcal{Y}) \mid U_{t} u \leq u, t \geq 0\right\} .
$$

The class of Lyapunov functions of RDS is a particular subclass of $\mathcal{S}_{\mathcal{U}}$ with some continuity properties [3]. The supermedian functions characterize globally the RDS behaviour. They are related with the so-called complete Lyapunov functions (see [8] for an overview).

\section{Hitting times}

Given a point $x \in \mathcal{Y}$, we define the first random hitting time of the random orbit of $x$ to the measurable subset $F \times$ $E \subset \Omega \times \mathcal{Y}$ by:

$$
T_{F \times E}(\omega, x):=\inf \left\{t>0 \mid \Phi_{t}(\omega, x) \in F \times E\right\} .
$$

If we fix $\omega \in \Omega$, we define the first random hitting time of the random orbit of $x$ to the measurable subset $E \subset \mathcal{Y}$ by:

$$
T_{E}^{\omega}(x):=T_{\Omega \times E}(\omega, x) .
$$

If the space $\mathcal{Y}$ is equipped with a metric $d$, we can define the first hitting time of $E$ as follows:

$$
T_{E}^{\omega}(x):=\inf \left\{t \geq 0 \mid d\left(\phi_{t}(\omega) x, E\right)=0\right\} .
$$

We define the first random exit time from $E$, denoted by $\zeta_{E}^{\omega}$ of the random orbit of $x$ to the measurable subset $E \subset \mathcal{Y}$ by $\zeta_{E}^{\omega}(x):=T_{\mathcal{Y} \backslash E}^{\omega}(x)$.

\section{RANDOM SAFETY PROBLEM}

In this section, we present the safety framework for RDS. The safety concepts are similar to those defined for stochastic processes [16], but we allow the randomization of the forbidden set. Then, some of these concepts become nonlinear.

\section{A. Safety concepts}

Let $S \subset \mathcal{Y}$ a measurable subset that represents the living state space of the $\operatorname{RDS}(\theta, \phi)$. Like in the previous sections, we denote the forbidden measurable set by $U$, which is a strict subset of $S$.

A point $x \in S$ is called strongly safe w.r.t. the unsafe set $U$, for an orbit $\omega$ starting in $x$, if $T_{U}^{\omega}(x)<\zeta_{S}^{\omega}(x)$. 
1) p-Safety: To make the safety definition more flexible, we need to consider the probability measure of the orbits starting in $x$. We define the concept of $\mathbf{p}$-safety (where $\mathbf{p} \in$ $[0,1)$ ) asking that this probability is dominated by $\mathbf{p}$.

A point $x \in S$ is called $\mathbf{p}$-safe if

$$
q(x):=\mathbb{P}\left\{\omega \mid T_{U}^{\omega}(x)<\zeta_{S}^{\omega}(x)\right\} \leq \mathbf{p} .
$$

This $\mathbf{p}$-safety is the direct generalization of the similar concept defined for Markov processes.

2) Random safety: In the context of RDS, all the definitions are presented in a randomized setting. Therefore the natural generalization is to consider safety for random sets. We will call the new concept random safety.

First we define the concept of random set [3]. Intuitively, random sets are set-valued random variables. They also can be thought of as representations of imprecise random variables.

We suppose that $\mathcal{Y}$ is a subset of the Euclidean space $\mathbb{R}^{d}$.

A function $\omega \mapsto M(\omega)$ taking values in the non-empty closed (respectively, compact) subsets of $\mathcal{Y}$ is called random closed (respectively, compact) set if

$$
\omega \mapsto d(x, M(\omega))
$$

is measurable for each $x \in \mathcal{Y}$, where

$$
d(x, M(\omega)):=\inf _{y \in M(\omega)} d(x, y) .
$$

A simple example of random closed set can be described as follows. Let $\xi$ be a random vector in the Euclidean space $\mathbb{R}^{d}$ and $\gamma$ a non-negative random variable. Then the random ball $B_{\gamma}(\xi)$ with radius $\gamma$ and centred in $\xi$ is a random closed set.

A function $\omega \mapsto U(\omega)$ is called random open set if $\omega \mapsto$ $U(\omega)^{c}$ is a random closed set.

A classical example of random set, can be defined as a level set. Suppose that $S$ is finite. Let $\psi: S \rightarrow[0,1]$ and $\alpha: \Omega \rightarrow[0,1]$ be a random variable uniformly distributed. Then define the random set $U: \Omega \rightarrow 2^{S}$ by

$$
U(\omega):=\{y \in S \mid \psi(y) \geq \alpha(\omega)\}
$$

Knowledge about an arbitrary random set $\Gamma$ is provided by the set of measurable selections (or, selectors):

$$
S(\Gamma):=\{\gamma: \Omega \rightarrow S \text { measurable } \mid \gamma(\omega) \in \Gamma(\omega), \forall \omega \in \Omega\} .
$$

Each selection $\gamma \in S(\Gamma)$ induces a probability distribution $\mathbb{P}_{\gamma}$ on the Borel $\sigma$-algebra of $S$ defined by:

$$
\mathbb{P}_{\gamma}(E):=\mathbb{P}\left[\left(\gamma^{-1}\right)(E)\right] .
$$

These probability distributions will have an essential role in the characterization of general distributions of random sets.

Let $S$ be a closed set and $U$ a random open set of $\mathcal{Y}$ with $U \subset S$. Then the concepts of safety are adapted straightforward to random forbidden sets. We may consider both closed and open random sets to model the forbidden set $U$. A simplified picture of random safety can be found in the Fig. 1.

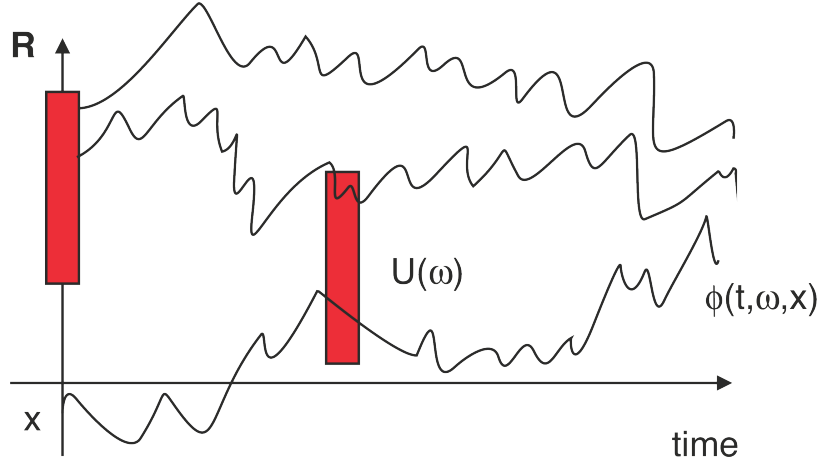

Fig. 1. Random safety. State-space is $S=\mathbb{R}$, and the unsafe set is a random set $U(\omega)$.

For each selector $\rho \in S(U)$ of the forbidden set, we can think the random safety as a reach avoidance problem for a probabilistic obstacle whose distribution is $\mathbb{P}_{\rho}$.

\section{B. Occupation and Hitting Measures}

We define the occupation measure for the complement (w.r.t. $S$ ) of the random open set $U$, denoted by $V$. By duality, we define the hitting measure associated to $U$. Note that both measures are nonlinear, but they are still connected with the occupation kernel as in the case of Markov processes.

In the theory of random sets, all information about a random closed (respectively, open) set is contained in its generalized probability distribution. This distribution is defined as the capacity hitting functional for random closed sets, and as capacity inclusion functional for random open sets.

For the random closed set $V$, some standard functional distributions can be defined [11]. The capacity functional $T_{V}(K)$, where $K$ is a compact set of $S$, of random set $V$ is defined as follows:

$$
T_{V}(K):=\mathbb{P}\{\omega \mid K \cap V(\omega) \neq \emptyset\} .
$$

Sometimes $T_{V}$ is called upper distribution of the random set $V$. The avoidance function is defined as $Q_{V}(K):=1-$ $T_{V}(K)$, i.e.,

$$
Q_{V}(K):=\mathbb{P}\left\{\omega \mid V(\omega) \subset K^{c}\right\} .
$$

It is possible to integrate with respect to the capacity functional. The Choquet integral of a non-negative measurable function is defined as in [11]:

$$
\int f d T_{V}:=\int_{0}^{\infty} T_{V}[f \geq s] d s=\mathbb{E} \sup _{v \in V} f(v) .
$$

Here, the Choquet integral is defined as a Riemann integral applied to the capacitary measure $T_{V}$ of variable level sets of $f$, i.e., applied to $g(s):=T_{V}(\{x \mid f(x) \geq s\})$.

When $K$ is a singleton, i.e. $K=\{x\}$ the capacity functional applied to this gives rise to the so-called covering function:

$$
p_{V}(x):=\mathbb{P}\{\omega \mid x \in V(\omega)\} .
$$


The covering function can also be interpreted as the expectation of the indicator function $I_{V}$ associated to the random set, i.e.,

$$
p_{V}(x)=\mathbb{E}\left[I_{V}(x)\right] .
$$

For a random open set $U$, the role of capacity functional is taking the inclusion functional:

$$
\mathcal{I}_{U}(F):=\mathbb{P}\{\omega \mid F \subset U(\omega)\}
$$

where $F$ is an arbitrary closed set of $S$. Sometimes, $\mathcal{I}_{U}$ is called also lower distribution of the random set $U$. In a similar way, according to [11], the Choquet integral with respect to $\mathcal{I}_{U}$ is defined as:

$$
\int f d \mathcal{I}_{U}:=\int_{0}^{\infty} \mathcal{I}_{U}[f \geq s] d s=\mathbb{E} \inf _{u \in U} f(u) .
$$

We define the following occupation measure of the closed random set $V$ corresponding to the $\operatorname{RDS}(\theta, \phi)$, as the covering function

$$
\mu_{V}^{\phi}(x):=\mathbb{E}\left[\sup _{t \geq 0} I_{V}\left(\phi_{t}(\cdot, x)\right)\right] .
$$

By duality, we define the hitting measure of the open random set $U$ corresponding to the $\operatorname{RDS}(\theta, \phi)$, as

$$
\nu_{U}^{\phi}(x):=\mathbb{E}\left[\inf _{t \geq 0} I_{U}\left(\phi_{t}(\cdot, x)\right)\right] .
$$

Let us consider the occupation measure of $S$, given by:

$$
G_{S}(x):=\mathbb{E} \int_{0}^{\zeta_{S}^{\omega}} \phi_{t}(\omega) x d t
$$

The evolution equation for the $\operatorname{RDS}(\theta, \phi)$ is

$$
G_{S}(x)=\mu_{V}^{\phi}(x)+\mathbb{E}\left[\inf _{t \geq T_{V}^{\omega}} I_{U}\left(\phi_{t}(\cdot, x)\right)\right] .
$$

The equation (20) may have resemblance with the analogous one existing for the case when the forbidden set is deterministic. The main problem in the random case is that the occupation and hitting measures are not additive. Therefore, the optimization techniques developed in [15] need to be extended for the nonlinear case.

\section{RANDOM BARRIER CERTIFICATES}

Let $S \subset \mathcal{Y}$ be the living space of the RDS $(\theta, \phi)$. Suppose now that an initial (measurable) set of states $A \subset S$ is given. The forbidden set $U$ is given as a random open set of $\mathcal{Y}$ with $U \subset S$. We use the notations, for a measurable function $h$ on $\mathcal{Y}$

$$
\begin{aligned}
H_{A} & :=\sup \{h(a) \mid a \in A\} \text { and } \\
H_{U} & :=\int h d \mathcal{I}_{U}:=\mathbb{E} \inf _{x \in U} h(x) .
\end{aligned}
$$

We will define barrier certificates as a subcone of the cone of supermedian functions.

Remark 1: If the forbidden set is modelled as a closed random set, then we define:

$$
H_{U}:=\int h d T_{U}:=\mathbb{E} \sup _{x \in U} h(x) .
$$

A function $h: \mathcal{Y} \rightarrow \mathbb{R}_{+}$is called a barrier function for the $\operatorname{RDS}(\theta, \phi)$ w.r.t. a triple $(A, U, S)$ if

- $h$ is supermedian on $S$, i.e.,

$$
h\left(\phi_{t}(\omega) x\right) \leq h(x), \forall x \in S, \omega \in \Omega, t \leq \zeta_{S} .
$$

- The separation condition holds:

$$
H_{A} \leq H_{U}
$$

Theorem 1: Let $(\theta, \phi)$ be an RDS with a given safety triplet $(A, U, S)$. Suppose that there exists a barrier function $h$ such that: $H_{A} \leq \mathbf{p}, H_{U} \geq 1$. Then the following probability bound holds:

$$
\mathbb{P}\left\{\omega \mid \exists t \leq \zeta_{S}(\omega) \text { s.t. } \phi_{t}(\omega) x \in U(\omega)\right\} \leq \mathbf{p}, \forall x \in A . \quad \text { (25) }
$$

Proof: Based on the fact that $H_{U} \geq 1$, we obtain the following inequality:

$$
\begin{array}{r}
\mathbb{P}\left\{\omega \mid \exists t \leq \zeta_{S}(\omega) \text { s.t. } \phi_{t}(\omega) x \in U(\omega)\right\} \leq \\
\mathbb{P}\left\{\sup _{t \in[0, \infty)} h\left(\phi_{t}(\omega) x\right) \geq 1\right\}, \forall x \in A .
\end{array}
$$

Applying the Markov's inequality for the random variable $\sup _{t \in[0, \infty)} h\left(\phi_{t}(\omega) x\right)$, and using the supermedian property of the barrier certificate, we obtain for all $x \in A$ :

$$
\begin{array}{r}
\mathbb{P}\left\{\sup _{t \in[0, \infty)} h\left(\phi_{t}(\omega) x\right) \geq 1\right\} \leq \\
\mathbb{E} \sup _{t \in[0, \infty)} h\left(\phi_{t}(\omega) x\right) \leq h(x) \leq H_{A} .
\end{array}
$$

The conclusion follows since $H_{A} \leq \mathbf{p}$.

In practice, the set of barrier functions of interest can be written as follows:

$\mathcal{C}_{b}(A, U, S):=\left\{h \in \mathcal{S}_{\mathcal{U}}(S) \mid H_{A}(h) \leq \mathbf{p}\right.$ and $\left.H_{U}(h) \geq 1\right\}$,

where $\mathcal{S}_{\mathcal{U}}(S)$ is the set of (nonrandom) supermedian functions on $S$.

The main novelty, comparing with the case when we deal with deterministic obstacles, in the definition of barrier certificates is the computation of $H_{U}$ as an integral w.r.t. the lower distribution of $U$. In practice, this condition has to be verified for each selector probability distribution of $U$.

In order to generate barrier certificates for RDS, one useful tool is provided by the stochastic Koopman operators. We call them stochastic and not random, since in the formula (2), we take the expectations.

Formally, the stochastic Koopman operators $K_{t}$ are defined on a space of measurable bounded functions (observables) $f: \mathcal{Y} \rightarrow \mathbb{R}$ for which the following functional exists:

$$
K_{t} f(x):=\mathbb{E}\left[f\left(\phi_{t}(\omega) x\right)\right], x \in \mathcal{Y} .
$$

The stochastic potential operator will be defined in a standard way:

$$
Q f(x):=\mathbb{E} \int_{0}^{\infty} f\left(\phi_{t}(\omega) x\right) d t, x \in \mathcal{Y} .
$$

Note that in the definition of the family of stochastic Koopman operators and its associated potential we apply the 
observables only to the cocycle part of RDS (not to the skew product as in the case of random Koopman operators).

Remark 2: 1) If $u: \mathcal{Y} \rightarrow \mathbb{R}_{+}$is supermedian with respect to the random Koopman semigroup, then

$$
K_{t} u \leq u .
$$

2) Based on the cocycle equations, it can be observed that $Q f$ is supermedian for all positive measurable bounded functions $f: \mathcal{Y} \rightarrow \mathbb{R}_{+}$.

It is obvious that barrier certificates are intimately connected with the Lyapunov functions that can be defined for RDS. Therefore, they can be generated using the eigenfunctions associated to the stochastic Koopman operators [9], but this topic will be investigated in a forthcoming paper. This approach will involve the approximation of stochastic Koopman operators using their spectrum [7].

In the following, we suppose that the associated stochastic Koopman operator family satisfies the semigroup property. This implies the Markov property of our RDS. This assumption is necessary to obtain more valuable characterizations of barrier certificates.

Define the generator of the stochastic Koopman family $\mathcal{K}=\left(K_{t}\right)_{t \geq 0}$ acting on the observable functions $f \in \mathcal{B}^{b}(\mathcal{Y})$ by the limit:

$$
\mathcal{L} f(x):=\lim _{t \searrow 0} \frac{K_{t} f(x)-f(x)}{t} .
$$

Using the Remark 2, we obtain the following result.

Proposition 1: If $\mathcal{K}=\left(K_{t}\right)_{t \geq 0}$ is an operator semigroup, then any barrier function $h$ is super-regular, i.e.,

$$
\mathcal{L} h \leq 0 .
$$

Remark 3: Note that the semigroup property is not sufficient to allow us to apply the barrier certificates characterizations for deterministic forbidden set obtained in [16] for Markov processes. The difficulty comes from the definition of $H_{U}$ as the integral with respect to a non-additive measure (functional capacity). Then the balayage methods for the super-regular functions do not apply in this case.

Theorem 2: The safety function $q$ is solution for the following Dirichlet problem:

$$
\int \mathcal{L} f d T_{V}=0
$$

where $V:=S \backslash U$, with boundary conditions:

$$
\int f d \mathcal{I}_{U}=1, f=0 \text { on } S^{c} .
$$

\section{Proof:}

The proof is the result of the representations of the Choquet integrals with respect to upper, respectively lower, distributions as supremum, respectively infimum of the integrals of the set of all measurable selections of the random sets $V$ and $U$.

Since, the space $\mathcal{Y}$ is Polish, applying Th.3.2 from [10], we obtain the following representations:

$$
\int \mathcal{L} f d T_{V}=\sup _{\gamma \in S(V)} \int \mathcal{L} f d \mathbb{P}_{\gamma}
$$

and

$$
\int f d \mathcal{I}_{U}=\inf _{\rho \in S(U)} \int f d \mathbb{P}_{\rho} .
$$

An RDS can be thought also a family of dynamical systems or Markov processes. Then for each $\omega \in \Omega$, the safety function is solution for the classical Dirichlet problem [16]. Then for two fixed selectors $\gamma \in S(V)$ and $\rho \in S(U)$, we have the corresponding safety function $q_{\gamma, \rho}$ characterized as solution for the following equation:

$$
\int \mathcal{L} f d \mathbb{P}_{\gamma}=0
$$

with boundary conditions:

$$
\int f d \mathbb{P}_{\rho}=1 \text { and } f=0 \text { on } S^{c} .
$$

Remark that the supports of $\mathbb{P}_{\gamma}$ and $\mathbb{P}_{\rho}$ are disjoint. Then the conclusion comes from the representations (32) and (33).

Remark 4: Theorem 2 shows that choosing appropriate measurable selectors for the random sets $U$ and $V$, one can find suitable approximations for the safety function. The selector sets are not convex, but they can be embedded in some convex cores as follows. The $\operatorname{core}\left(\mathcal{I}_{U}\right)$ is the set of all countably additive probability measures that setwise dominate this functional. The $\operatorname{core}\left(T_{V}\right)$ is the set of all countably additive probability measures that setwise are dominated by $T_{V}$. The sets of selectors are weak*-closed convex hull in their cores [5]. In this way, it is possible to obtain upper and lower bounds of the Choquet integrals, which appear in the above theorem.

\section{BARRIER CERTIFICATES FOR PARTICULAR RDS}

\section{A. Random differential equations}

Let $\left(\Omega, \mathcal{F}, \mathbb{P}, \theta_{t}\right)$ be a metric dynamical systems. We consider a continuous-time RDS generated by the random differential equation (RDE) of the following form:

$$
\dot{x}=F\left(\theta_{t}(\omega), x\right),
$$

defined on the space $\mathcal{Y} \subset \mathbb{R}^{d}$. Note that here, the randomness is regarding the parameters that do not depend the system state. The RDS generated by the RDE (34) is defined by:

$$
\phi_{t}(\omega) x=x+\int_{0}^{t} F\left(\theta_{s}(\omega), \phi_{s}(\omega) x\right) d s .
$$

This kind of RDS under different regularity assumptions on $F$ has been studied in [1]. The solution of the RDE (34) with initial condition $\phi_{0}(\omega) x=x$ is thought of as a family of random variables, which are the trajectories $\phi_{t}(\omega) x$ starting in $x$. Because the RDE solutions are defined pathwise, for each fixed $\omega$, the RDE (34) is, in fact, an ordinary differential equation. If the solution of the RDE (34) is differentiable with respect to $t$ and the family of stochastic Koopman operators is a semigroup, then the generator of $\left(K_{t}\right)$ has the following expression:

$$
\mathcal{L} f:=\mathbb{E}[F(\omega, x)] \nabla f(x), \forall f \in C_{b}^{1}\left(\mathbb{R}^{d}\right) .
$$


This result can be found in [7]. The barrier certificates for such an RDS can be generated using the eigenfunctions of the generator (36).

\section{B. Diffusion processes as RDS}

Let $\Omega:=\left\{\omega: \mathbb{R} \rightarrow \mathbb{R}^{d} \mid \omega\right.$ is continuous and $\left.\omega(0)=0\right\}$. Let $\mathcal{F}:=\mathcal{B}(\Omega)$ be the Borel $\sigma$-algebra of $\Omega$. Denote the Wiener process (Brownian motion) by $W_{t}: \Omega \rightarrow \mathbb{R}^{d}$, given by $W_{t}(\omega):=\omega(t)$. For $t \in \mathbb{R}$, define the Wiener shift $\theta_{t}$ : $\Omega \rightarrow \Omega$ by:

$$
\theta_{t} \omega(s):=\omega(s+t)-\omega(t) .
$$

Then, $(\Omega, \mathcal{F}, \mathbb{P}, \theta)$ is a metric dynamical system.

Let us consider the standard stochastic differential equation $(\mathrm{SDE})$ :

$$
d X_{t}=b\left(X_{t}\right) d t+\sigma\left(X_{t}\right) d W_{t}, X_{0}(\omega)=x, t \in \mathbb{R}
$$

where $b: \mathbb{R}^{d} \rightarrow \mathbb{R}^{d}$ and $\sigma: \mathbb{R}^{d} \rightarrow \mathbb{R}^{d} \times \mathbb{R}^{d}$ are such that

$$
|b(x)-b(y)|+|\sigma(x)-\sigma(y)| \leq D|x-y|, \forall x, y \in \mathbb{R}^{d},
$$

for some constant $D>0$.

Then (38) admits a unique solution (called diffusion process):

$$
\phi_{t}(\omega) x=x+\int_{0}^{t} b\left(\phi_{s}(\omega) x\right) d s+\int_{0}^{t} \sigma\left(\phi_{s}(\omega) x\right) d W_{s}(\omega)
$$

where $\int_{0}^{t} \sigma\left(\phi_{s}(\omega) x\right) d W_{s}(\omega)$ is the standard stochastic integral w.r.t. the Wiener process. The generator of the stochastic Koopman generator has the following expression:

$$
\mathcal{L} f(x)=b(x) \nabla f(x)+\frac{1}{2} \operatorname{Tr}\left(\sigma(x)\left(\nabla^{2} f(x)\right) \sigma(x)^{\top}\right)
$$

where $\operatorname{Tr}$ denotes the trace of the matrix.

We have studied barrier certificates for SDEs in [16], when the forbidden set is supposed to be deterministic. When the forbidden set is random, the Dirichlet problem characterization of the safety function becomes nonlinear.

\section{Markov chains as RDS}

Markov chain are related to i.i.d. RDS. Their connection has become a classical topic in the specialised literature (see, e.g., [1], [17]). We do not elaborate the topic here. We only mention that, for control Markov chains, the reach avoidance problem with random obstacles with applications in air traffic management has been studied in [14].

\section{CONCLUSIONS}

In this paper, we have studied safety of a random dynamical system, precisely the reach-avoidance problem with a random obstacle. We have provided characterizations of random safety using occupation and hitting measures and Koopman operators. The main difficulty that we had to overcome is that the randomness of the obstacle set leads to some nonlinear constraints in the definition of barrier certificates. Therefore, the Dirichlet problem characterization of barrier certificates is nonlinear, in the sense we need to consider the integration with respect to upper or lower distributions of random sets.

Furthermore, in this paper, we have opened new research avenues for obtaining data-driven approximations of barrier certificates by applying data-driven techniques for the Koopman operators [7]. The techniques developed in this paper will be used in a follow-up paper for studying the problem of ship capsizing, when the ship dynamics is modelled as an RDS.

\section{ACKNOWLEDGEMENTS}

The first and third authors acknowledge the support from MSRC sponsors DNV-GL, Royal Caribbean Cruise Ltd. The second author was partly supported by the Poul Due Jensen's Foundation in the project SWIft.

\section{REFERENCES}

[1] Arnold, L.: Random Dynamical Systems. Springer Monographs in Mathematics (1998).

[2] Arnold, L., Chueshov, I., Ochs, G.: Random Dynamical Systems Methods in Ship Stability: A Case Study. In: Deuschel JD., Greven A. (eds) Interacting Stochastic Systems. Springer, Berlin, Heidelberg (2005)

[3] Arnold, L., Schhmalfuss, B.: Lyapunov's second method for RDS. Journal of Differential Equations 177, (2001): 235-265.

[4] Manuela L. Bujorianu, Rafael Wisniewski, Evangelos Boulougouris. Stochastic Safety for Markov Chains. IEEE Control Systems Letters, Vol 5(2), April 2021.

[5] Castaldo, A., MacCheroni, F., Marinacci, M.: Random Correspondences as Bundles of Random Variables. The Indian Journal of Statistics, Vol 66(3) (2004): 409-427.

[6] Chen, Y.; Singletary, A.W.' Ames, A.D.: Density Functions for Guaranteed Safety on Robotic Systems. 2020 American Control Conference (ACC), Denver, CO, USA, (2020): 3199-3204.

[7] Crnjaric-Žic, N., Macešic, S. Mezic, I.: Koopman Operator Spectrum for Random Dynamical Systems. J. Nonlinear Science Springer (2019).

[8] Giesl, P., Hafstein, S.: Review on computational methods for Lyapunov functions. Discrete and Continuous Dynamical Systems - Series B, 20 (8), (2015); 2291-2331.

[9] Mauroy, A., Mezić, I, Susuki, Y.: The Koopman Operator in Systems and Control - Concepts, Methodologies, and Applications. LNCIS, volume 484 (2020).

[10] Miranda, E., Couso, I., Gil, P.: Random sets as imprecise random variables. J. Math. Anal. Appl. 307 (2005): 32-47.

[11] Molchanov, I., Theory of Random Sets. Probability and Its Applications. Springer (2017).

[12] Prajna, S., Jadbabaie, A., Pappas, G. J.: A Framework for WorstCase and Stochastic Safety Verification Using Barrier Certificates. IEEE Transactions on Automatic Control 52(8) (2007): 1415-1428.

[13] Saperstone, S. H.: Semidynamical systems in infinite dimensional spaces. Springer Verlag (1981).

[14] Summers, S., Kamgarpour, M., Lygeros, J., Tomlin, C.: A stochastic reach-avoid problem with random obstacles. Proc. ACM 14th International Conference on Hybrid Systems: Computation and Control. (2011): 251 - 260.

[15] Wisniewski, R., Bujorianu, M.L., Sloth, C.: p-Safe Analysis of Stochastic Hybrid Processes. IEEE Transactions on Automatic Control, to appear (2020).

[16] Wisniewski, R., Bujorianu, M.L.: Safety of Stochastic Systems: An Analytic and Computational Approach. Submitted.

[17] Ye, F. X.-F., Wang, Y., Qian, H.: Stochastic dynamics: Markov chains and random transformations. Discrete and Continuous Dynamical Systems - Series B. 21 (7) (2016): 2337-2361.

[18] Vorob'ov, O.Y., Random set models of fire spread. Fire Technology vol. 32, (1996): 137-173.

[19] Xu, X., Tabuada, P., Grizzle, J.W., Ames, A.D.: Robustness of Control Barrier Functions for Safety Critical Control, IFAC-PapersOnLine, vol. 48(27), 2015: 54-61. 